Polyisobutylen von $P=2600(M=145000)$ in Benzol angeführt ${ }^{20}$, was etwa $3 \% /{ }^{\circ} \mathrm{C}$ entspricht, also recht erheblich ist.

Eine gute Bestätigung der erwarteten gegenläufigen Abhängigkeit von [ $\eta]$ und $T K$ gibt Tab. 5 nach Evans und Young ${ }^{31}$, die Polyisobutylen (ähnlich B 15) in verschiedenen Lösungsmitteln bei 2 Temperaturen gemessen haben. Das zuletzt aufgeführte Lösungsmittel ist als Ester nicht ohne weiteres mit den Kohlenwasserstoffen vergleichbar.

Die Bestätigung der Erwartung nach Abb. 1 ist künftigen Untersuchungen vorbehalten.

Esं sei hier nur erwähnt, daß „Mizellkolloide“ vielfach kleinere TK als „Molekülkolloide" haben,

${ }^{31}$ H. C. Evans u. D. W. Y o ung, Ind. Engng. Chem. 34, 461 [1942]; 39, 1676 [1947]. somit kann der $T K$ nicht als entscheidende Größe für eine Unterscheidung beider Stoffklassen benutzt werden (vgl. Anm. 2).

Aus den besprochenen Ergebnissen geht deutlich hervor, welch große Bedeutung die eingehende Untersuchung der Beeinflussung der Viskositätszahl durch Lösungsmittel und Temperatur für das Verständnis des Zustandes der Hochmolekularen in Lösung hat. Diese Erscheinungen sind aber bisher eher als Störungen betrachtet worden.

Nach Abschluß der Arbeit sind dem Verf. folgende Arbeiten zugänglich geworden, die zum Teil sehr ähnliche Gedankengänge entwickeln: M. L. H u g g i n s, J. appl. Physics 10, 700 [1939]; 14, 246 [1943]; P. J. F 1 o r y , J. chem. Physics 10, 51 [1942]; T. A l f r e y, A. B a r o v i c s und H. M a r k, J. Amer. chem. Soc. 64, 1557 [1942] (zitiert nach Huggins II). Diese Autoren führen die Lösungsmittelabhängigkeit der Viskositätszahl auf Änderungen der Knäuelung zurück, da eine starke Wechselwirkung des Gelösten mit dem Lösungsmittel eine gestrecktere Form des Fadenmoleküls und eine höhere Viskositätszahl bedingt.

\title{
Kohlenoxydverbindungen der Nichtmetalle II
}

\author{
Eigenschaften von Carbonylcyanid \\ Von Oskar Glemser und Viktor HäUsser ${ }^{1}$ \\ Aus dem Institut für anorganische Chemie und Elektrochemie der Rheinisch- \\ Westfälischen Technischen Hochschule Aachen \\ (Z. Naturforschg. 3b, 159-163 [1948]; eingegangen am 24. April 1948)
}

\begin{abstract}
Carbonylcyanid wird nach M a la chowski und Mitarbb. ${ }^{2}$ dargestellt. Das im Hochvakuum gereinigte Produkt schmilzt bei $-37,9_{5}^{\circ}{ }^{\circ}$, die Dampfdrucke folgen der Tensionsgleichung $\log p=-1960 / T+8,6679$; Sdp. ${ }_{760} 65,6_{8}^{\circ}$; mittlere molare Verdampfungswärme 8969 cal; Troutonsche Konstante 26,5. Für die Flüssigkeitsdichten zwischen 0 und $1 \delta, 6_{0}^{\circ}$ ist die lineare Beziehung $d_{t}=1,1541(1-0,001211 t)$ gültig. Der Brechungsindex ist $n_{\mathrm{D}}^{18,40}=1,3547$; Molekularrefraktion 15,45 ; Dielektrizitätskonstante $\varepsilon_{18,40}=10,68$; Dipolmoment $\mu=1,35 \cdot 10^{-18}$ el.st. E. Molare Verbrennungswärme bei konstantem Druck $331,97 \pm 0,64 \mathrm{Kcal}$; Bildungswärme nach $3 \mathrm{C}_{\beta-\mathrm{Graph}}+1 / 2 \mathrm{O}_{2}+\mathrm{N}_{2}=\mathrm{CO}(\mathrm{CN})_{2}-47,5 \mathrm{Kcal}$. Die Löslichkeit in Phosgen bei $-22,8$ und $0^{\circ}$ sowie die Löslichkeit in Carbonylselenid bei $-31,3_{0}{ }^{\circ}$ wird mittels Dampfdruckmessungen bestimmt.

Durch Belichtung wird Carbonylcyanid nicht verändert; gegenüber trockenen porösen Stoffen bleibt es auch bei $100^{\circ}$ beständig.
\end{abstract}

$\mathrm{D}$ ie erste erfolgreiche Synthese des Carbonylcyanids $\mathrm{CO}(\mathrm{CN})_{2}$ gelang $\mathrm{Mal} \mathrm{achowski}$ und Mitarbb. ${ }^{2}$. Die vorher darüber angestellten Versuche sind praktisch ohne Ergebnis geblieben. So vermochten weder $\mathrm{Carstanjan}$ und $\mathrm{Schertel^{4 }}$ aus Quecksilbercyanid und Kohlenoxyd, noch Gintl ${ }^{5}$ aus Silbercyanid und Phosgen, Carbonylcyanid zu gewinnen. Auch ist es sehr zweifelhaft, ob Berthelot und Ga u de chon ${ }^{6}$ aus Kohlenoxyd und Dicyan bei der Einwirkung stiller elektrischer Entladungen oder durch ultraviolette Strahlen ein polymeres

1 Diss. V. Häusser, Techn. Hochschule Aachen 1943.

2 R. M a l a chowski, L. Jurkiewicz u. J. W o j t o w i c z, Ber. dtsch. chem. Ges. 70, 1012 [1937].

3 Alle Temperaturangaben in Celsius-Graden.
Carbonyleyanid erhalten haben. D i e ls und Mitarbb.7, die durch Ozonisierung von Oxymethylenmalonnitril die Synthese versuchten, kamen ebenfalls nicht zum Ziel.

Malachowski und Mitarbb. ${ }^{2}$ benützten als Ausgangsmaterial Diisonitrosoaceton, das mit Essigsäureanhydrid in das Diacetylderivat übergeführt wurde. Durch thermische Abspaltung von 2 Mol. Essigsäure bildete sich Carbonylcyanid.

${ }^{4}$ Carstanjan u. Schertel, J. prakt. Chem. 4, 49 [1871].

5 Gintl, J. prakt. Chem. 4, 362 [1871].

${ }^{6}$ D. B e r thelot u. H. Gaud e ch on, C. R. hebd. Séances Acad. Sci. 156, 1766, 1990 [1913].

7 O. Diels, H. Gärtner u. R. Kn a a ck, Ber. dtsch. chem. Ges. 55, 3439 [1922]. 


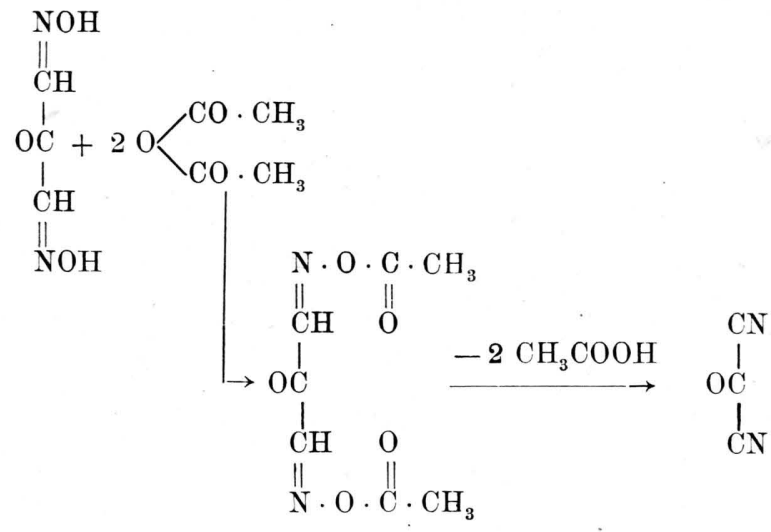

Carbonylcyanid wird als klare Flüssigkeit vom Sdp. $65,5^{\circ}$ und Schmp. $35-36^{\circ}$ beschrieben; spezif. Gewicht bei $20^{\circ}=1,124$, Brechungsindex $n_{\mathrm{D}}^{20}=1,3919$. Es brennt mit purpurvioletter, blaugesäumter Flamme und erinnert in seinem Geruch an Blausäure. Mit kaltem Wasser reagiert es explosionsartig unter Bildung von Cyanwasserstoff und Kohlensäure.

Carbonylcyanid verdient Beachtung, weil es sich als ein wirksames Mittel zur Einführung des Cyanformylrestes in organische Verbindungen erwies ${ }^{8}$. Uns interessierte die Beziehung des Carbonylcyanides zu den Carbonylhalogeniden; ein Vergleich mit dem von uns näher untersuchten Carbonylselenid ${ }^{9}$ lag nahe.

\section{Darstellung von Carbonyleyanid}

Wir hielten uns bei der Darstellung von $\mathrm{CO}(\mathrm{CN})_{2}$ im wesentlichen an die Vorschrift von Malachowski und Mitarbb.2. Bezogen auf Diisonitrosoaceton, konnten wir eine Ausbeute von $40 \%$ erreichen (Literaturangabe $44 \%$ ). Zu beachten ist, daß von der Nitrosierung der Acetondicarbonsäure an bis zur Darstellung des Carbonylcyanids alle Reaktionen als Nebenprodukt erhebliche Mengen Blausäure lieferten ${ }^{\mathbf{1 0}}$.

Als Verunreinigungen waren Blausäure, Kohlendioxyd und Essigsäure vorhanden. Das Rohprodukt wurde mehrmals fraktioniert und dann in einer Hochvakuumapparatur aus Jenaer Glas bei verschiedenen Drucken bis zur Konstanz der Dampfdrucke destilliert.

Die explosionsartig verlaufende Hydrolyse bei Zugabe von Wasser konnten wir bestätigen. Die bei langsamer Hydrolyse entstehende feste Zwischenverbindung, vermutlich freie Cyanameisensäure ${ }^{2}$, isolierten wir im Hochvakuum. Sie schmilzt im Hochvakuum zwischen 35,5 und $36,5^{\circ}$ unter Zersetzung; bei Zimmertemperatur zersetzt sie sich binnen 15 bis 20 Min. in $\mathrm{CO}_{2}$ und $\mathrm{HCN}$.

\section{II. $\mathrm{E}$ i g e n s ch a f te $\mathrm{n}$}

Dampfdrucke: Als temperaturkonstante Bäder wurden Tetrachlorkohlenstoff, Wasser und Eisessig bei

8 DRP. 666394.

9 O. G le ms e r u. T. R is le r, Z. Naturforschg. 3 b, 1 [1948].

\begin{tabular}{|c|c|c|c|c|c|}
\hline$t^{0}$ & $-22, \mathbf{8}_{\mathbf{8}}$ & 0,0 & $\mathbf{8 , 3 _ { 5 }}$ & $13,0_{0}$ & $\mathbf{1 7 , \mathbf { 5 } _ { \mathbf { 0 } }}$ \\
\hline $\begin{array}{c}p_{\mathrm{mm} \mathrm{Hg}} \\
\text { gef. }\end{array}$ & 6,8 & 30,8 & $\mathbf{5 0 , 3 _ { 5 }}$ & $6 \mathbf{5 , 4}$ & $83, \mathbf{8}_{\mathbf{5}}$ \\
\hline $\begin{array}{c}p_{\mathrm{mm} \mathrm{Hg}} \\
\text { ber. }\end{array}$ & 6,8 & 30,8 & $\mathbf{5 0 , 3 _ { \mathbf { 6 } }}$ & 65,4 & $83, \mathbf{5}$ \\
\hline
\end{tabular}

Tab. 1. Dampfdruckwerte von $\mathrm{CO}(\mathrm{CN})_{2}$.

ihren Schmelztemperaturen benützt; die Temperaturen von 13,0 und $17,5_{0}^{\circ}$ wurden mit einem HöpplerUltrathermostaten konstant gehalten. Zur Temperaturbestimmung dienten Dampfdruckthermometer nach Stock ${ }^{11}$ und geeichte Thermometer; die Ablesung des Meniskus erfolgte mit einem Kathetometer auf $0,1 \mathrm{~mm}$ genau. Die Dampfdruckwerte werden durch die Tensionsgleichung $\log p=-1960 / T+8,6679$ wiedergegeben. Daraus errechnete sich der Siedepunkt zu $65,68^{\circ}$ bei $760 \mathrm{~mm} \mathrm{Hg}$; die mittlere molare Verdampfungswärme betrug 8969 cal. Die Troutonsche Konstante ergab sich zu 26,5.

Der Schmelzpunkt wurde nach Stock ${ }^{12}$ im Hochvakuum bestimmt; Mittelwert aus 3 Bestimmungen: $-37,9_{5} \pm 0,05^{\circ}$.

Molekulargewicht aus der Dampfdichte: $0,12423 \mathrm{~g}$ $\mathrm{CO}(\mathrm{CN})_{2}$ wurden in einem Glaskolben von $651,94 \mathrm{~cm}^{3}$ bei $16,0^{\circ}$ (Höppler-Ultrathermostat) verdampft; im Kolben herrschte danach ein Druck von $43 \mathrm{~mm} \mathrm{Hg}$. Daraus ermitteltes Molekulargewicht 79,90, theoretischer Wert 80,02. Bei Zimmertemperatur liegt dampfförmiges $\mathrm{CO}(\mathrm{CN})_{2}$ monomolekular vor.

Flüssigkeitsdichte ${ }^{13}$ : Als Pyknometer wurde eine $6 \mathrm{~cm}$ lange, durch einen piceingedichteten Schliff mit der Hochvakuumapparatur verbundene Präzisionskapillare benutzt. $1 \mathrm{~cm}$ der Kapillare hatte das Volumen 0,01199 $\mathrm{cm}^{3}$; das Volumen des Pyknometers bis zu einer Marke wurde durch Auswägen mit Quecksilber zu $0,2786 \mathrm{~cm}^{3}$ bei $22,0^{\circ}$ bestimmt. Die Umrechnung dieses Volumens auf die Versuchstemperaturen wurde nach $v_{t}=0,2786[1-(22,0-t) / 40000]$ vorgenommen. Bei den Versuchstemperaturen 12,0 und $18,6_{0}{ }^{\circ}$ wurde mit einem Dewar-Gefäß, das mit Sichtstreifen versehen war, gearbeitet; ein Höppler-Ultrathermostat hielt das Bad konstant. Als weitere Versuchstemperatur wurde noch $0^{\circ}$ (Eiswasser) gewählt. Die im Pyknometer befindliche Substanz wurde in einer Glaskirsche kondensiert und die Menge an $\mathrm{CO}(\mathrm{CN})_{2}$ durch Ein- und Auswaage des Glases bestimmt. Im Temperaturbereich von $0,0^{\circ}$ bis $18,6^{\circ}$ ist für die Dichten folgende Gleichung gültig (s. Tab. 2).

Molekularrefraktion: Reines $\mathrm{CO}(\mathrm{CN})_{2}$ kann wegen seines kleinen Brechungsindexes nicht im Pulfrich-

10 Näheres über die Darstellung s. bei V. Häus $\mathrm{se}^{1}$.

11 A. S t o c k, Ber. dtsch. chem. Ges. 54, 1119 [1921]; Z. Elektrochem. angew. physik. Chem. 29, 355 [1923]. 12 A. S t o c k, Ber. dtsch. chem. Ges. 50, 156 [1917].

13 E. Wi berg u. W. Math ing, Ber. dtsch. chem. Ges. 70, 690 [1937]. 


\begin{tabular}{|c|c|c|c|c|c|}
\hline \multicolumn{6}{|c|}{$d_{t}=1,1541(1-0,001211 \cdot t)$} \\
\hline Temp. ${ }^{\circ} \mathrm{C}$ & $\begin{array}{c}\text { Flüssig- } \\
\text { keitsvolu- } \\
\text { menin cm }\end{array}$ & $\begin{array}{c}\text { Gewicht } \\
\text { in g }\end{array}$ & $\begin{array}{c}\mathrm{CO}(\mathrm{CN})_{2}- \\
\text { Gas in g }\end{array}$ & $\begin{array}{c}\text { Dichte } \\
\text { gef. }\end{array}$ & $\begin{array}{c}\text { Dichte } \\
\text { ber. }\end{array}$ \\
\hline$-0,0_{5}$ & 0,27795 & 0,32081 & 0,00031 & 1,1542 & 1,1534 \\
$12,0_{0}$ & 0,28189 & 0,32050 & 0,00062 & 1,1370 & 1,1374 \\
$18,6_{0}$ & 0,28375 & 0,32024 & 0,00088 & 1,1286 & 1,1286 \\
\hline
\end{tabular}

Tab. 2. Flüssigkeitsdichten von $\mathrm{CO}(\mathrm{CN})_{2}$.

Refraktometer untersucht werden. Infolgedessen wurde der Brechungsindex einer Benzollösung, deren Dichte vorher ermittelt worden war, gemessen und daraus nach der Mischungsregel von $\mathrm{Roth}$ und $\mathrm{E}$ ise $\mathrm{n}$ lo hr ${ }^{14}$ der des $\mathrm{CO}(\mathrm{CN})_{2}$ berechnet ${ }^{15}$. Die strenge Additivität der Eigenschaften von Lösungsmittel und Gelöstem ist bezüglich der Brechungsindices bis zu einer Konzentration von etwa 0,3 Mol Substanz in

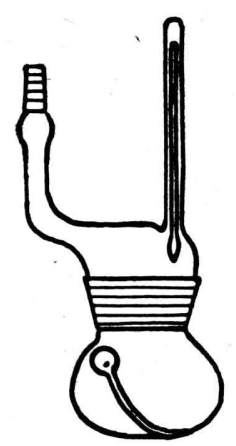

Abb. 1.

Zertrümmerungsapparat. 1 Mol Lösungsmittel vorhanden, was Rumscheidt16 in einer ausführlichen Untersuchung von Terpenen in Benzol festgestellt hat.

Bestimmung der Dichte des Gemisches: In eine Glaskirsche destilliert man $\mathrm{CO}(\mathrm{CN})_{2}$, schmilzt ab, wägt die Kirsche und bringt sie danach in den in Abb. 1 gezeichneten Zertrümmerungsapparat. Durch das Gewicht des fallenden Glasstabes, der magnetisch hochgehoben werden kann, wird die Halsspitze der Kirsche, die einseitig angeritzt ist, ohne nennenswerte Splitterbildung glatt abgeschlagen. $\mathrm{CO}(\mathrm{CN})_{2}$ destilliert nun in das mit flüssiger Luft gekühlte Pyknometer. Das Pyknometer wird mit Benzol aufgefüllt und die Flüssigkeitsdichte der Mischung, wie bereits beschrieben, bei $18,4_{0}{ }^{\circ}$ bestimmt. Zur Verwendung kam über Natrium getrocknetes Benzol (p. a. Merck cryst.), das zur weiteren Reinigung noch im Hochvakuum destilliert worden war. Nach der Dichtebestimmung destillierte man das tief gelbgrüne Benzol-CO $(\mathrm{CN})_{2}$-Gemisch in eine Glaskirsche mit Schliffansatz von bekanntem Gewicht, schmolz ab und wog. Die Dichte des Benzols bei $18,4_{0}{ }^{\circ}$ wurde nach $\mathrm{Lug}$ in in ${ }^{\mathbf{1 7}}$, der

14 W. A. Roth u. E. Eisenlohr, Refraktometr. Hilfsbuch, 1911.

15 Nach $\frac{n^{2}-1}{n^{2}-2} \cdot \frac{100}{d p}-\frac{n^{2}-1}{n^{2}-2} \cdot \frac{100-p}{d_{2} p} M=R$; $d=$ Dichte des Gemisches; $d_{2}=$ Dichte des Benzols; $p=$ Gew. $\% \mathrm{CO}(\mathrm{CN})_{2} ; 100-p=$ Gew.-\% Benzol; $M=$ Molekulargewicht des $\mathrm{CO}(\mathrm{CN})_{2}$.

16 Unveröff. Mitt. von Prof. Dr. P. Lip p, Aachen. $17 \mathrm{Lu} \mathrm{g}$ i n i n, Ann. Chimie 11, 465 [1867].

$18 \mathrm{~W}$ e e g m a n $\mathrm{n}$, Z. physik. Chem. 2, 237 [1888].

19 Hersteller $\mathrm{H}$ a a r d t u. Cie., Düsseldorf.
Brechungsindex des Benzols bei $18,4_{0}^{\circ}$ nach We e g m a $n n^{18}$ berechnet.

\begin{tabular}{|c|c|c|c|c|c|}
\hline $\begin{array}{c}\mathrm{CO}(\mathrm{CN})_{2} \\
\mathrm{~g}\end{array}$ & $\begin{array}{c}\text { Benzol } \\
\mathrm{g}\end{array}$ & $\begin{array}{c}\text { Gew.- } \% \\
\mathrm{CO}(\mathrm{CN})_{2}\end{array}$ & $V_{\text {fl. }}$ & $\begin{array}{c}\text { Gemisch } \\
\text { in gasf. }\end{array}$ & Dichte \\
\hline 0,04857 & 0,24075 & $\mathbf{1 6 , 7 8 _ { 8 }}$ & 0,31859 & 0,00073 & 0,9058 \\
\hline
\end{tabular}

Tab. 3. Dichte eines Benzol-CO(CN) $)_{2}$-Gemisches; Temp. des Refraktometers $18,4_{0}{ }^{\circ}$.

Brechungsindex des Gemisches: 1,4765.

Molekularrefraktion $R_{18,40}^{\mathrm{D}}=15,45$; berechnet 15,46 . Brechungsindex $\mathrm{CO}(\mathrm{CN})_{2} \quad n_{\mathrm{D}}^{\mathbf{1 8 , 4 0}}=1,3547$.

Dielektrizitätskonstante: Man bestimmte die Kapazität einer Benzollösung von $\mathrm{CO}(\mathrm{CN})_{2}$ und von Benzol (p. a. Merck cryst., im Hochvakúum dest.). Zur Anwendung kam das Dielkometer ${ }^{19}$. In einer etwa $10 \mathrm{~cm}^{3}$ großen Ampulle mit Schliffansatz von bekanntem Gewicht wurde im Hochvakuum etwas Benzol bei $-40^{\circ}$ kondensiert. Dazu destillierte man - wie unter Molekularrefraktion beschrieben - eine bekannte Menge $\mathrm{CO}(\mathrm{CN})_{2}$ hinzu, schmolz ab und erhielt aus der Auswaage die. Menge Benzol in der Mischung. Der über Phosphorpentoxyd getrocknete Kondensator wurde mit der Lösung gefüllt und bei $18,4_{0}{ }^{\circ}$ die Messung durchgeführt. Die Dielektrizitätskonstante der $\mathrm{CO}(\mathrm{CN})_{2^{-}}$ Benzol-Mischung ergab sich zu $\varepsilon_{18,40}=2,39$; der Prozentgehalt an $\mathrm{CO}(\mathrm{CN})_{2}$ betrug $4,5 \mathrm{Gew} . \%$. Die molare Polarisation berechnet sich danach $\mathrm{zu} 54,14^{20}$ für $\mathrm{CO}(\mathrm{CN})_{2}$ und daraus die Dielektrizitätskonstante $\varepsilon_{18,40}=10,68$. Nach der Formel von Debye beträgt dann das Dipolmoment $\mu=1,35 \cdot 10^{-18}$ elektrostat. Einheiten.

Verbrennungswärme ${ }^{21}$ : Verbrennungswärmen von Substanzen, die unter $100^{\circ}$ sieden, sind wegen Fehlzündungen und Verrußungen in der kalorimetrischen Bombe nur schwierig zu bestimmen. Durch einen kleinen Kunstgriff haben wir für $\mathrm{CO}(\mathrm{CN})_{2}$ die erwähnten Fehlermöglichkeiten praktisch ausgeschaltet.

Zur Wasserbestimmung diente Benzoesäure p.a. als Eichsubstanz, die vorher 2 Stdn. bei $60^{\circ}$ getrocknet worden war. Die Zündung erfolgte durch einen $3,5 \mathrm{~mm}$ starken Platindraht. Die bei der Verbrennung entstehende Salpetersäure aus dem Stickstoff der Sauerstoffbombe wurde nach Titration mit ${ }^{1 / 14,9}-n . \mathrm{Na}_{2} \mathrm{CO}_{3}$ Lösung und Kongorot als Indikator mit $0,2 \mathrm{cal}$ in Rechnung gesetzt. Auf Vor- und Nachperiode entfielen je 6 Ablesungen in der Minute. ${ }^{20}$ Aus $P_{2}=\frac{P-f_{1} P_{1}}{f_{2}} ; P=$ Polarisation des Ge-
misches; $P_{1}=$ Polarisation des Benzols; $P_{2}=$ Polarisation von $\mathrm{CO}(\mathrm{CN})_{2} ; \boldsymbol{f}_{\mathbf{1}}=$ Molenbruch des Benzols; $f_{2}=$ Molenbruch des $\mathrm{CO}(\mathrm{CN})_{2}$.

21 Die verwendete Vaseline und der Baumwollfaden, beide von bekannter Verbrennungswärme, sind uns freundlicherweise von Hrn. Prof. Dr. W. A. Roth, Freiburg i. Brg., zur Verfügung gestellt worden, wofür wir auch an dieser Stelle unseren herzlichsten Dank aussprechen. 
Verbrennung des Carbonylcyanids: Nach einem Vorschlag von W. A. R oth ${ }^{22}$ wurde die Substanz in einer zugeschmolzenen Glaskirsche verbrannt, die, wie Abb. 2 zeigt, für unsere Verhältnisse eine besondere Form erhielt. Am Bauch der Kirsche befanden sich zwei dünnwandige, warzenförmige Ausbuchtungen, die mit Vaseline bekannter Verbrennungswärme bestrichen wurden und durch einen Baumwollfaden mit dem Zünddraht verbunden waren. Die Kirsche hing am Platindraht im üblichen Quarzschälchen. In diese Glaskirsche bekannten Gewichts, die zunächst mit Schliffansatz versehen war, wurde bis zum Ansatz der beiden Warzen im Hochvakuum $\mathrm{CO}(\mathrm{CN})_{2}$ destilliert und unter Kühlung mit flüssiger Luft der Halsansatz möglichst kurz zugeschmolzen und zu einem kleinen Hals ausgezogen. Nach der Verbrennung war eine geringe Rußabscheidung in der Glaskirsche zu beobachten, die bei der Auswertung berücksichtigt wurde ${ }^{22}$. Nach dem Öffnen der Bombe wurde geprüft, ob kein HCN-Geruch festzustellen war.

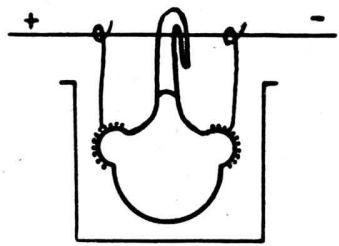

Abb. 2. Glaskirsche

zur Bestimmung der Verbrennungswärme.

Folgende Daten wurden benützt:

Verbrennungswärme von Benzoesäure . . 6324,5 cal Verbrennungswärme von Vaseline . . . . 11048 cal Verbrennungswärme des Baumwollfadens 389 cal.

\begin{tabular}{|c|c|c|c|c|c|c|}
\hline $\begin{array}{c}\text { Ein- } \\
\text { waage } \\
\mathrm{g}\end{array}$ & $\begin{array}{c}\mathrm{Ruß} \\
\mathrm{g}\end{array}$ & $\begin{array}{c}\text { Vase- } \\
\text { line } \\
\text { in cal }\end{array}$ & $\begin{array}{c}\text { Faden } \\
\text { in cal }\end{array}$ & $\begin{array}{c}\text { Zün- } \\
\text { dung } \\
\text { cal }\end{array}$ & $\begin{array}{c}\text { Salpe- } \\
\text { tersäure } \\
\text { cal }\end{array}$ & $\begin{array}{c}\text { Verbr.- } \\
\text { Wärme } \\
\text { cal/g }\end{array}$ \\
\hline 0,4184 & 0,0031 & 314,86 & 22,17 & $\mathbf{1 2 , 9 4}$ & 9,3 & $\mathbf{4 1 4 6 , 6}$ \\
0,3542 & 0,0019 & 361,27 & $\mathbf{1 9 , 4 5}$ & $\mathbf{9 , 5 4}$ & 9,1 & $\mathbf{4 1 6 2 , 6}$ \\
\hline
\end{tabular}

Tab. 4. Bestimmung der Verbrennungswärme.

Die Verbrennungswärme von $\mathrm{CO}(\mathrm{CN})_{2}$ bei konstantem Volumen ist $4154,6 \mathrm{cal} / \mathrm{g} \pm 8,0$ cal. Daraus berechnete molare Verbrennungswärme bei konstantem Druck: $331,97 \pm 0,64 \mathrm{Kcal}$. Die Bildungswärme ist $-47,5$ Kcal nach

$$
3 \mathrm{C}_{\beta \text {-Graph }}+\frac{1}{2} \mathrm{O}_{2}+\mathrm{N}_{2}=\mathrm{CO}(\mathrm{CN})_{2}-47,5 \mathrm{Kcal}^{23} \text {. }
$$

Löslichkeit von $\mathrm{CO}(\mathrm{CN})_{2}$ in $\mathrm{COCl}_{2}$ : In ein Wägegefäß nach $\mathrm{Stoc \textrm {k } ^ { 1 2 }}$ wurde eine bestimmte Menge $\mathrm{CO}(\mathrm{CN})_{2}$ im Hochvakuum gebracht und gewogen. Dann wurde im Hochvakuum (bis zur Konstanz der Dampfdrucke) gereinigtes Phosgen in kleinen Anteilen hinzudestilliert und nach jeder Einwaage von

22 W. A. R oth, Thermochemie, Berlin 1932.

23 J. C h i p m a n, Ind. Engng. Chem. 24, 1013 [1932].
$\mathrm{COCl}_{2}$ der Dampfdruck der Mischung bei 0,0 und $-22,8_{8}^{\circ}$ bestimmt. Die in Tab. 5 angegebenen Dampfdrucke sind Mittelwerte aus drei Ablesungen.

\begin{tabular}{|c|c|c|c|c|c|c|c|}
\hline \multicolumn{8}{|c|}{ A. Temperatur: $0,0^{\circ}$} \\
\hline $\begin{array}{c}\mathrm{CO}(\mathrm{CN})_{2} \\
\mathrm{Mol-} \%\end{array}$ & 76,25 & 50,71 & 50,39 & 35,56 & 26,67 & 13,31 & 0,0 \\
\hline $\boldsymbol{p}_{\mathrm{mm} \mathrm{Hg}}$ & $153,5_{5}$ & $316,3_{5}$ & $303,2_{0}$ & $393,4_{0}$ & 443,6 o & $501,4_{0}$ & $556,8_{0}$ \\
\hline \multicolumn{8}{|c|}{ B. Temperatur: $-22,8_{8}{ }^{\circ}$} \\
\hline $\begin{array}{c}\mathrm{CO}(\mathrm{CN})_{2} \\
\mathrm{Mol}-\%\end{array}$ & 76,25 & 50,71 & 50,39 & 35,56 & 26,67 & 13,31 & 0,0 \\
\hline$p_{\mathrm{mm} \mathrm{Hg}}$ & $78,9_{5}$ & $131,1_{5}$ & $131,1_{0}$ & $151,1_{0}$ & $162,0_{0}$ & $178,1_{0}$ & $196,4_{0}$ \\
\hline
\end{tabular}

Tab. 5. Löslichkeit von $\mathrm{CO}(\mathrm{CN})_{2}$ in $\mathrm{COCl}_{2}$.

Löslichkeit von $\mathrm{CO}(\mathrm{CN})_{2}$ in COSe: Nach derselben Methodik, wie eben beschrieben, wurde die Löslichkeit von $\mathrm{CO}(\mathrm{CN})_{2}$ in COSe bestimmt. In Tab. 6 sind die Mol-Proz. $\mathrm{CO}(\mathrm{CN})_{2}$ und die dazugehörigen Dampfdrucke bei $-31,3_{0}{ }^{\circ}$ eingetragen; auch hier sind die angegebenen Dampfdrucke Mittelwerte aus $3 \mathrm{Ab}$ lesungen.

\begin{tabular}{|c|c|c|c|c|c|c|}
\hline \multicolumn{6}{|c|}{ Temperatur: $-31,3_{0}{ }^{\circ}$} \\
\hline $\begin{array}{c}\mathrm{CO}(\mathrm{CN})_{2} \\
\mathrm{Mol}-\%_{0}\end{array}$ & 96,68 & 74,71 & 57,46 & 42,95 & 36,03 & 0,0 \\
\hline$p_{\mathrm{mm} \mathrm{Hg}}$ & $23,5_{5}$ & $181,5_{5}$ & $342,1_{0}$ & $431,4_{0}$ & $450,2_{5}$ & 498,1 \\
\hline
\end{tabular}

Tab. 6. Löslichkeit von $\mathrm{CO}(\mathrm{CN})_{2}$ in COSe.

Einwirkung von Licht auf $\mathrm{CO}(\mathrm{CN})_{2}$ : Ungefiltertes Quecksilberdampflicht sowie monochromatische Strahlung (Wellenlänge $5700 \AA$ ) haben auf Carbonylcyaniddampf und flüssiges Carbonylcyanid keinerlei Einfluß. Im Quecksilberdampflicht erscheint das flüssige Carbonylcyanid tief gelbgrün gefärbt, während bei gewöhnlichem Licht eine ganz schwache Gelbfärbung nur bei größeren Mengen in der Durchsicht $\mathrm{zu}$ erkennen ist.

Das der Bestrahlung ausgesetzte Carbonylcyanid befand sich in durchsichtigen Quarzgefäßen, die an die Hochvakuumapparatur angeschlossen werden konnten. Der Eintritt einer möglichen Reaktion wurde durch Dampfdruckmessungen verfolgt. In beiden Fällen, bei Bestrahlung mit ungefiltertem Quecksilberdampflicht, wie bei Bestrahlung mit Licht der Wellenlänge $5700 \AA$, blieb der Dampfdruck des Carbonylcyanids unverändert.

Verhalten gegen poröse Stoffe: An wasserfreien porösen Stoffen, z. B. wasserfreier Aktivkohle, wird $\mathrm{CO}(\mathrm{CN})_{2}$ nicht zersetzt, auch nicht in Gegenwart von trockener Luft, wie Tensionsmessungen zeigten. Es wird beim Erwärmen auf $100^{\circ}$ unverändert von der Kohle wieder abgegeben. An feuchter Aktivkohle da- 
gegen ist vollständige $\mathrm{Hydrolyse}$ in $\mathrm{HCN}$ und $\mathrm{CO}_{2} \mathrm{zu}$ beobachten.

Vergleicht man Carbonylcyanid mit den Carbonylhalogeniden und -chalkogeniden, dann kann ausgesagt werden: Gemeinsam ist die leichte Hydrolysierbarkeit; bei Carbonylcyanid ist diese Eigenschaft so stark ausgeprägt, daß es mit Wasser explosionsartig reagiert. Carbonylcyanid unterscheidet sich aber von diesen Verbindungen durch seine stark negative Bildungswärme (vgl. Tab.6), von den Carbonylhalogeniden, auch Carbonylselenid, durch seine Beständigkeit gegenüber Licht. Bemerkenswert ist seine Nichtspaltbarkeit in Kohlenoxyd und Dicyan unter den beschriebenen Bedingungen.

Im dampfförmigen Zustand ist $\mathrm{CO}(\mathrm{CN})_{2}$ monomolekular, im flüssigen Zustand sind Abweichungen vom Theorem der übereinstimmenden Zustände vorhanden.

\begin{tabular}{|l|l|c|c|c|}
\hline & Schmelzp. & Sdp. & $\begin{array}{c}\text { Verdampfungs- } \\
\text { entropie } \\
L_{s} / T_{s}\end{array}$ & $\begin{array}{c}\text { Bildungs- } \\
\text { enthalpie } \\
\text { Kcal/Mol }\end{array}$ \\
\hline $\mathrm{COF}_{2}$ & $-114,0$ & $-83,1$ & 20,3 & - \\
$\mathrm{COCl}_{2}$ & -126 & 8 & 21,3 & $-\overline{53,5}$ \\
$\mathrm{COBr}_{2}$ & -93 & $63-64$ & 21,5 & $-1,0$ \\
$\mathrm{COS}$ & $-138,85$ & $-59,2$ & 22,6 & -35 \\
$\mathrm{COSe}$ & $-124,4$ & $-21,7$ & 21,0 & - \\
$\mathrm{CO}(\mathrm{CN})_{2}$ & $-37,95$ & 65,2 & 26,5 & 47,5 \\
\hline
\end{tabular}

Tab. 7. Vergleich von $\mathrm{CO}(\mathrm{CN})_{2}$ mit Carbonylhalogeniden und -chalkogeniden.

Hrn. Prof. Dr. A. B en ath, dem ehemaligen Direktor des Instituts, sind wir für wohlwollende Unterstützung zu Dank verpflichtet.

\title{
Über Chalkone II"
}

\section{Die bakteriostatische Wirkung natürlicher und synthetischer Chalkone}

\section{Von Ernst Schraufstätter** und Sigismund Deutsch}

(Z. Naturforschg. 3 b, 163-171 [1948]; eingegangen am 1. März 1948)

\begin{abstract}
Ausgehend von dem Befunde, daß Butein (2'.4'.3.4-Tetraoxy-chalkon) eine deutliche bakteriostatische Wirksamkeit bei Staph.aureus erkennen ließ, wurde eine gröfiere Anzahl synthetischer Chalkone untersucht. Mehrere dieser Verbindungen hemmten das W'achstum von Staph. aureus in erheblicher Verdünnung, während Salmonella paratyphi nur in geringem Maße beeinflußt wurde. Durch Serumzusatz wurde die Wirkung der untersuchten Verbindungen weitgehend abgeschwächt.
\end{abstract}

$\mathrm{V}$ on natürlichen und synthetischen Derivaten des Chalkons, Flavanons, Flavons und Flavonols wurden bisher lediglich von $\mathrm{Mahal}^{\mathbf{1}}$ einige wenige Flavone, darunter das Chrysin, bei Typhus- und Colibakterien untersucht. Diese Verbindungen beeinflußten jedoch die Entwicklung der genannten Bakterien nicht.1a

Die antibiotischen Eigenschaften verschiedener ungesättigter Ketone und Lactone sind bereits bekannt.<smiles>C[C@@H]1CCCCO1</smiles>

Parasorbinsäure

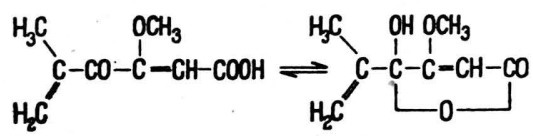

Penicillinsäure
* I. Mitt.: E. S ch raufstätter u. S. Deutsch, Chem. Ber., im Druck.

** Erlangen, Schuhstraße 19.

1 H. S. M a h a 1, Proc. Indian Acad. Sci. Sect. B, 5, 186 [1937], ref. in Chem. Zbl. 1938 I, 1397.

1a Nach Fertigstellung dieser Arbeit wurden uns Publikationen von J. N a g h s ki, M. J. C o p le y u. J. F. C o u ch, Science [New York] 105, 125 [1947], und A. A. Andersen u. J. A. B e r r y, Science [New York] 106, 644 [1947], bekannt, in denen über die bakteriostatische Wirkung des Flavonolderivats Quercetin berichtet wird. Auch ist inzwischen eine
Die von $\mathrm{Kuhn}$ und Mitarbb.2 synthetisierte Parasorbinsäure (in der Natur in Vogelbeeren vorkommend) hemmte in der Verdünnung 1: 2000 das Wachstum von Staph.aureus und von Hefe. Durch Hydrierung der Doppelbindung geht die Wirkung vollkommen verloren. Die aus dem Kulturfiltrat verschiedener Penicillium-Arten isolierte Penicillinsäure zeigte noch in einer Verdünnung von 1:50000 bei Staph. aureus und $E$. coli einen bakteriostatischen Effekt ${ }^{3}$. Völlig inaktiv erwies sich dagegen die Dihydropenicillinsäure. Das ebenfalls von mehreren Penicillien gebildete Patulin unterdrückt in einer Verdünnung 1:64000 das Wachstum von Staph. aureus noch völlig.

Arbeit von D. H. Marrian, P. B. Russellu. A. R. T o d d, J. chem. Soc. [London] 1947, 1419, über Aminochalkone erschienen. Die bakteriostatischen Wirkungen dieser Aminoderivate des Chalkons waren jedoch nur sehr geringfügig. Ferner untersuchten kürzlich F. B lank u. R. Sut e r, Experientia 4, 72 [1948], Anthocyane und Flavonole auf ihr Verhalten gegenüber verschiedenen Mikroorganismen.

${ }_{2}$ R. K uln, D. Jerchel, F. Moew us u. E. F. M öl l e r, Naturwiss. 31, 468 [1943].

${ }_{3}$ H. Raistrick, A. E. Oxford u. G. Smith, Chem. and Ind. 61, 22 [1942]. 